\title{
Does Pregabalin Change Serum Levels of iNOS and NO in Diabetic Patients With Neuropathic Pain? A Quasi-experimental Study
}

Moosareza Memari

Ardebil University of Medical Sciences

Mirsaber Sadat Amini

Shahid Beheshti University of Medical Sciences School of Medicine

Yalda Khazaei Poul

Shahid Beheshti University of Medical Sciences School of Medicine

Khojasteh Joharchi ( $\square$ joharchi-kh@sbmu.ac.ir)

Shahid Beheshti University of Medical Sciences School of Medicine https://orcid.org/0000-0001-81468200

Research article

Keywords: Diabetic Peripheral Neuropathic Pain (DPNP), Inducible Nitric Oxide Synthase (iNOS), Nitric Oxide (NO), Pregabalin (PGB)

Posted Date: August 3rd, 2020

DOl: https://doi.org/10.21203/rs.3.rs-28062/v1

License: (c) (1) This work is licensed under a Creative Commons Attribution 4.0 International License. Read Full License 


\section{Abstract \\ Background}

Pregabalin (PGB) has been approved for the treatment of diabetic peripheral neuropathic pain (DPNP), but the mechanism of the PGB effect in this situation is not precisely known. Would it be via the reduction of inducible Nitric Oxide Synthase (iNOS) and thus the decrease of Nitric Oxide (NO) in type 2 diabetic patients? The current clinical trial was conducted to answer this question.

\section{Methods}

Twenty Seven diabetic patients with DPNP > 4, assessed by Visual Analogue Scale (VAS), were enrolled in this study. They received placebo/Bd for ten days as a washout period, then a starting dose of $75 \mathrm{mg} / \mathrm{Bd}$ PGB for one week and $150 \mathrm{mg} / \mathrm{Bd}$ for the next seven weeks. We took a fasting blood sample at baseline, before starting the treatment (BT) with PBG, then one month after the treatment (OMT), and also at the end of two months (TMT). The iNOS and NO serum levels were measured using the ELISA kit and the Griess method, respectively.

\section{Results}

Significant time-dependent reduction of iNOS of serum and DPNP intensities observed $(P<0.05)$. However, the serum levels of NO reduced significantly in OMT compared to BT $(P<0.05)$, but no significant differences were seen between OMT and TMT $(P>0.05)$.

\section{Conclusions}

Our study revealed a direct correlation between serum levels of iNOS and NO with the treatment of DPNP by PGB, thus introducing a possible mechanism for pain-relieving properties of PGB.

\section{Trial registration:}

This study was approved by the ethics committee of our University of Medical Sciences, (ethical code: IR.SBMU.MSP.REC.1395.41) and registered in our Registry of Clinical Trials (at 14/03/2016) and approved in 19/07/2017, the registration number is: (IRCT2017012932277N1). The study was conducted in the Diabetes Clinic of a teaching Hospital on 22/07/2017 till 18/03/2018. We have explained the study objectives and protocols to the subjects and obtained written informed consent before they participated in the study.

\section{Background}


Several pathologic mechanisms are reported for diabetic neuropathy (DN) and diabetic peripheral neuropathic pain (DPNP). The increment of inducible Nitric Oxide Synthase (iNOS) is one of the possible pivotal mechanisms of DPNP. Overproduction of NO following iNOS expression plays an essential role in the pathogenesis of DPNP $(1,2)$. In higher concentrations, NO is neurotoxic to the local neural tissue via peroxynitrite production, leading to the deterioration of regional circulation and inhibiting the axonal mitochondria through oxidative modifications of the mitochondrial proteins of cells $(3,4)$. Other studies proved the effect of iNOS and NO in the plasticity development of neuropathic pain $(5,6)$. Indirect inhibition of iNOS by Alpha-lipoic acid (7) and direct inhibition by $N^{G}$-nitro-L-Arginine Methyl Ester ( $L$ NAME) and some other natural and synthetic iNOS inhibitors attenuated nociceptive hypersensitivity associated with inflammatory and neuropathic pain models in animals (8-11).

DPNP causes sleep disorders, persons' morale dissatisfaction, and annoying life. The loss of proprioception in advanced stages of DN leads to diabetic foot and consequently amputation in advanced stages of DN $(12,13)$.

PGB is one of the three drugs which have been approved by the Food and Drug Administration (FDA) for DPNP treatment. The mechanism of action of PGB in this situation is not fully understood, and the inhibition of voltage-gated calcium channels is the most likely proposed mechanism $(14,15)$. Some studies are showing the reduction effect of PGB on the iNOS and NO in some animal tissues at various pathologic conditions such as osteoarthritis (16), brain ischemia (17), diabetes (18), and epilepsy (19).

In our previous study (20) we found that PGB is effective and well-tolerated in our patients with DPNP But as far as we searched there is no clinical trial about the effect of PGB on serum concentration of iNOS and NO in diabetic patients. Thus, to clarify the mechanism of drug action and to find the new targets for pharmacotherapy, it is necessary to perform clinical trials besides the relevant animal studies. Consequently, the current study was conducted to evaluate the effects of PGB on serum concentrations of iNOS and NO in type II diabetic patients and to explain the role of these mediators modulation in the treatment of DPNP

\section{Methods}

\section{Ethics approval and consent to participate}

This study was approved by the ethics committee of our University of Medical Sciences, (ethical code: IR.SBMU.MSP.REC.1395.41) and registered in our Registry of Clinical Trials website: (at 14/03/2016) and approved in 19/07/2017, the registration number is: (IRCT2017012932277N1). The study was conducted in the Diabetes Clinic of a teaching Hospital on 22/07/2017 till 18/03/2018. We have explained the study objectives and protocols to the subjects and obtained written informed consent before they participated in the study.

\section{Patients and Study design}


In this quasi-experimental study, we chose a total of twenty-seven type-II diabetic patients from our diabetes clinic, considering some inclusion and exclusion criteria. The study was conducted from July 2017 to March 2018 in compliance with the Helsinki declaration. The understudy patients' diabetes and their diabetic peripheral neuropathy were diagnosed by a specialized endocrinologist using American Diabetes Association Guideline 2017 (21) and Michigan Neuropathy Screening Instrument (MNSI) (22) respectively. Subjects were randomly screened and selected according to inclusion and exclusion criteria.

The inclusion criteria were as follows: 1- having type-Il diabetes for more than 5 years; 2- aged $40-$ 65 years old; 3- having MNSI examination score of more than two; 4- DPNP intensity at least more than 40 millimeters by Visual Analogue Scale (VAS) and 6 months duration; 5- no smoking; 6- no intake of synthetic or herbal drugs for DPNP treatment at least 15 days before the beginning of the study; 7- not using Nitrates containing medicines and Sildenafil; 8- not using synthetic or botanical antioxidants.

The exclusion criteria were: 1- emerging any intolerable adverse drug reaction due to PGB; 2- any neuropathy and pain other than DPNP such as arthritis, gout, surgery, hyperuricemia; 3 - developing any acute illnesses such as an infection; 4- having chronic diseases including severe hepatic, renal and cardiovascular disorders; 5 - any changes in the kind and dosage of medications, diet, and physical activities during of the study.

Because of human rights, we could not have a control group, and our ethical committee did not approve a group of patients having pain and do not receive any drug for an extended period of two months. Thus the patients received PGB placebo capsules twice/day only for10 days as a washout period and $75 \mathrm{mg} / \mathrm{Bd}$ PGB capsule in the first week of treatment, followed by $150 \mathrm{mg} / \mathrm{Bd}$ for seven more weeks. Neither physicians nor the patients were aware of the contents of the capsules. We took the fasting blood samples of the subjects before and after treatment in three-time courses. The first time was before treatment (BT), or at the baseline (after the washout period and before the administration of $75 \mathrm{mg}$ PGB. The second sample was taken one month after treatment (OMT), or four weeks later in the middle of the study, and third: eight weeks later or two months after treatment (TMT), at the end of the study.

\section{DPNP assessment}

The Endocrinologist measured the intensity of patients' pain by the VAS method, and we calculated the monthly mean of patients' pain at three times: BT, OMT, and TMT. Many previous studies approved this method for patients' pain measuring. The absence of pain is expressed by zero, and the worst pain is stated 100 millimeters on the ruler. The numbers also represent the pain between these two ranges according to the patient's feelings (23).

\section{Biochemical assays}

\section{Serum iNOS concentration measurement}

The three obtained fasting blood samples of each participant were allowed to clot for 10-15 minutes at room temperature, and then serums were separated by centrifuging (Beckman Avanti J-25; Beckman 
Coulter, Brea, CA, USA) at $3000 \mathrm{rpm}$ for 10 minutes. Immediately after centrifugation, we stored the supernatant serums at $-70^{\circ} \mathrm{C}$ until iNOS assay. Before the beginning of the analysis, we kept the frozen samples at room temperature (RT) for at least 30 minutes. Then we assessed the concentrations of iNOS in serum samples by applying a commercial enzyme-linked immune sorbent assay (ELISA) kit (ZellBio $\mathrm{GmbH}$, Germany, Cat. No: ZB-10928S-H9648). This kit is based on the Biotin double antibody sandwich technology to assay the human iNOS. The wells of microplates of the package are pre-coated with an anti-iNOS monoclonal antibody. After adding the serum, iNOS of serum adheres to the pre-coated monoclonal antibody. After that, adding anti-iNOS antibody labeled with biotin to combine streptavidinHRP leading to form an immune complex. Multi-step washing processes removed unbound enzymes. Immune complex plus chromogen solutions created the color, which the related optic density is proportional to the concentration of the iNOS. After adding a stop solution, we measured the optic density of unknown amounts of serum iNOS of patients at $450 \mathrm{~nm}$ wavelength using the ELISA reader (BioTek Instruments, Inc., Winooski, VT, USA). Finally, we calculated the iNOS concentrations of serum samples using the standard curve.

\section{Serum NO concentration measurement}

Blood samples of each participant were allowed to clot for 10-15 minutes at room temperature, and then serums were separated by centrifuging (Beckman Avanti J-25; Beckman Coulter, Brea, CA, USA) at $3000 \mathrm{rpm}$ for 20 minutes. Then the supernatant serums were stored at $-70^{\circ} \mathrm{C}$ until NO assay via applying a kit based on the Griess colorimetric method (ZellBio GmbH, Germany, Cat. No: ZB-NO-96A).

After gradually defrosting the samples in RT, they were first deproteinized and then assessed by the use of the Griess Method in a 96 flat microplate. We prepared the standard solutions of Nitrate and Nitrite in a serial dilution. We performed the reduction of nitrate to nitrite by adding vanadium-(III)-chloride $(8 \mathrm{mg} / \mathrm{ml})$. The Griess reagent was made of $50 \mu \mathrm{L}$ sulfanilamide (2\%) and $50 \mu \mathrm{L} \mathrm{N}$-(1-Naphthyl) ethylenediamine dihydrochloride ( $0.1 \%)$. After adding Griess reagent to samples, they were incubated at $37^{\circ} \mathrm{C}$ for 30 minutes, and after color formation, the absorbance was observed at $540 \mathrm{~nm}$ wavelength using the ELISA reader (BioTek Instruments, Inc., Winooski, VT, USA). As a final point, the NO concentrations were calculated using the standard curve.

\section{Statistical analysis}

We analyzed the data using the SPSS 19 . We used the Kolmogorov-Smirnov test was to assess the normality of the data. We used the Repeated measure method and paired t-test to detect the effect of intervention and time on iNOS, NO, and DPNP. To assess the correlation of iNOS, NO, and DPNP, we used the Pearson correlation test. We considered the results to be statistically significant at a p-value of less than 0.05 .

\section{Results}

\section{The pain Scores:}


The consort flow chart of our study is presented in Fig. 1. Ninety-five male and female patients were randomly interviewed and examined, and fifty-nine of them were excluded using exclusion criteria. Thirtysix patients were included and given a placebo for ten days as a washout period. After the first blood sampling (BT) and during the first month, five patients left the study: three patients because of the unpleasant adverse drug reactions (ADRs) and two for ineffectiveness. One month after the beginning of treatment (OMT), in second-time points, three other subjects, and at the end of the study, in third-time points, one more subject was not available for blood sampling. Lastly, three samples of twenty-seven patients were used for biochemical assays.

However, all of the study subjects were included in the final statistical analysis according to the intentionto-treat principle. General characteristics and mean baseline values of primary outcomes are presented in Table 1.

Table 1

Demographic and Mean \pm SD clinical characteristics of included patients $(N=27)$

\begin{tabular}{ll} 
Characteristics & Values \\
\hline Both Genders $(\mathrm{N})$ & 27 \\
Male $(\mathrm{N})$ & 7 \\
Female $(\mathrm{N})$ & 20 \\
Age $(\mathrm{Yrs})$ & $54.52 \pm 6.18$ \\
BMI $\left(\mathrm{kg} / \mathrm{m}^{2}\right)$ & $26.5 \pm 2.2$ \\
HA1c $(\mathrm{mg} \%)$ & $8.24 \pm 1.25$ \\
FBS (mg/dl) & $152.04 \pm 21.64$ \\
MNSI questionnaire & $6.71 \pm 2.03$ \\
MNSI examination & $2.82 \pm 0.43$ \\
VAS (mm) & $6.81 \pm 1.08$ \\
Serum Creatinine (mg/dl) & $1.02 \pm 0.11$ \\
Duration of Diabetes(Yrs.) & $8.2 \pm 3.2$ \\
Duration of DPNP (Yrs.) & $3.5 \pm 1.6$
\end{tabular}

Note: N: number of patients that completed the study; Yrs: years; BMI: Body Mass Index; HA1c: Hemoglobin A1c;

FBS: Fasting Blood Sugar; MNSI; Michigan Neuropathy Scale Instrument; VAS: Visual Analogue Scale

In the Pearson correlation model (Table 2) serum levels of iNOS and NO were significant predictors of DPNP intensity as measured by VAS before treatment $(P<0.05)$. 
Table 2

The correlation of baseline values of VAS, iNOS and NO

\begin{tabular}{|lllll|}
\hline Variables & & VAS & iNOS & NO \\
\hline VAS & $r$ & - & 0.397 & 0.516 \\
\cline { 2 - 5 } & $\mathrm{p}$-value & - & 0.040 & 0.006 \\
\hline iNOS & $\mathrm{r}$ & 0.397 & - & 0.651 \\
\cline { 2 - 4 } & $\mathrm{p}$-value & 0.040 & - & $<0.001$ \\
\hline NO & $\mathrm{r}$ & 0.516 & 0.651 & - \\
\cline { 2 - 4 } & $\mathrm{p}$-value & 0.006 & $<0.001$ & \\
\hline $\begin{array}{l}\text { Data analysis was done by Pearson correlation. VAS: visual analog scale; iNOS: inducible nitric oxide } \\
\text { synthase; NO: nitric oxide }\end{array}$ & & \\
\hline
\end{tabular}

According to the categorized DPNP scores, at the baseline (BT), all of the study subjects had moderate to severe pain scores, while at the second (OMT) and third (TMT) time points $88.9 \%$ and $100 \%$ of the study subjects were located in the mild pain category (Table 3 ).

Table 3

Pain severity during the study period according to categorized DPNP scores

\begin{tabular}{|c|c|c|c|c|c|}
\hline \multicolumn{2}{|c|}{ Variable } & \multicolumn{3}{|c|}{ Timepoints } & \multirow[t]{2}{*}{ P-value } \\
\hline & & BT & OMT & TMT & \\
\hline \multirow[t]{3}{*}{ Pain } & Mild & $0(0)$ & $24(88.9)$ & $27(100)$ & \multirow[t]{3}{*}{$<0.001$} \\
\hline & Moderate & $12(44.4)$ & 3 (11.1) & $0(0)$ & \\
\hline & severe & 15 (55.6) & $0(0)$ & $0(0)$ & \\
\hline \multicolumn{6}{|c|}{$\begin{array}{l}\text { P-value was reported based on Friedman test. Mild }=\text { DPNP }<4 \text {; Moderate }=4 \leq \text { DPNP }<7 \text {; Severe }=7 \leq \\
\text { DPNP }\end{array}$} \\
\hline
\end{tabular}

On the other hand, the mean \pm SE of VAS scores at the baseline, middle, and the end of the study were $6.82 \pm 0.23,2.85 \pm 0.12$, and $1.33 \pm 0.12$ millimeters, respectively. Thus according to the results obtained from the Repeated Measure Test, a significant time-dependent reduction of pain was observed (Fig. 2).

\section{The iNOS serum levels:}

The study results showed that the mean serum level of iNOS reduced time-dependently $(P<0.05)($ Fig. 3). The administration of PGB for one month significantly decreased the Mean $\pm S E$ serum level of iNOS from 
$152.37 \pm 11.15$ to $133.81 \pm 9.92 \mathrm{U} / \mathrm{L}$ (Unit/Liter). Moreover, after two months, it cut to $119.89 \pm 9.76 \mathrm{U} / \mathrm{L}$, which was also significantly lower than BT and OMT $(P<0.05)$ (Fig. 3$)$.

\section{The NO serum levels:}

Although the NO serum level was reduced during the study period (Fig. 4), unlike the iNOS, this reduction was not in a time-dependent manner. In BT, OMT, and TMT the Mean \pm SE serum levels of NO were $82.48 \pm$ $4.78,70.15 \pm 3.73$, and $66.10 \pm 4.41 \mu \mathrm{M} / \mathrm{L}$ (micromole/Liter), respectively. Even though the serum levels of NO were significantly reduced in OMT and TMT in comparison to the baseline value $(P<0.05)$, but no significant differences were seen between OMT and TMT ( $P>0.05)$ (Fig. 4).

\section{Discussion}

In this quasi-experimental study, we examined the effect of PGB on pain scores in type-2diabetic patients with DPNP and tried to understand the relation of serum concentrations of iNOS and NO in this process. Our first finding is showing the time-dependent reduction of pain following the PGB administration (Fig. 2). This finding is consistent with some similar studies in the field of neuropathic pain $(24,25)$. PGB alleviates the various types of neuropathic pain and presents itself as a first-line therapeutic agent with remarkable safety and efficacy. Many clinical studies in different age groups and different types of neuropathic pain have projected that PGB is the most effective agent either in monotherapy or in combined regimens in terms of cost-effectiveness, tolerability, and an overall improvement in neuropathic pain states $(24,25)$.

On the other hand, we observed the time-dependently decrement of iNOS following PGB treatment (Fig. 3), and also a positive correlation of serum levels of iNOS and DPNP intensity (Table 2). This correlation is in line with Purwata et al. study which their results indicated that the iNOS and NO production by macrophages in diabetic patients with DPNP are 1.5 times more than that in neuropathic diabetic patients without DPNP (1).

Our results confirmed that the decrement of iNOS following PGB treatment results in NO reduction (Fig. 4), which is also in correlation with the lessening of DPNP (Table 2). The iNOS regards as a significant contributor to the initiation and exacerbation of degenerative conditions in both central and peripheral nervous systems after the overproduction of NO. This NO, in combination with superoxide, will result in peroxynitrite generation and nitrosylation of mitochondrial metabolic enzymes, leading to neuronal cell death. In a diabetic state, the overproduction of NO is seen and is related to the increase of iNOS production $(26,27)$. It has been shown that the essential molecule for the gene transcription and mRNA stability of iNOS is NF-KB. The role of NF-KB in inflammatory and neurodegenerative procedures has been well known $(17,28,29)$. Thus we can propose that the decrement of NO and iNOS in our study by PGB can be attributable to inhibition of the NF-KB transcription pathway. Further studies would clarify this hypothesis. 
There is other substantial evidence that the activation of iNOS and the overproduction of NO and the following nitrosative stress are involved in the development of DPNP. One of this evidence is the reduction of DPNP in diabetic Wistar rats by S-methylisothiourea sulfate as a specific inhibitor of iNOS (30). In some other indications, the increase of iNOS in neuropathic pain models such as pain caused by the herpes virus (31), the sciatic nerve chronic constriction injury $(\mathrm{CCl})(11,32)$ and the segmental spinal ligation of lumbar (Chung model of pain) in animals (33), have also been shown.

Furthermore, we have shown in our previous study (24), and it has also been demonstrated in many other studies that synthetic and natural inhibitors of iNOS will reduce the pathological levels of NO in different tissues of animals $(9,10,32-36)$. These studies which have been performed on various models of neuropathic pain have shown that neuropathic pain decreases along with inhibition of iNOS activity after administration of iNOS inhibitors $(9,10,24,29,31-34)$. It has been revealed that synthetic iNOS inhibitors such as $N^{G}$-nitro-L-arginine methyl ester (L-NAME), $N^{G}$-monomethyl-L-arginine (L-NMMA), and also1400W can reduce neuropathic pain (32-34). Likewise, the KD7332 as an iNOS dimerization inhibitor was effective in the formalin model of nociception and Chung model of neuropathic pain (10). Similarly, other studies have exposed that the use of Sulforaphane derived from Broccoli (30) and Capnellene extracted from a soft Coral (9) in the $\mathrm{CCl}$ model of neuropathic pain can treat neuropathic pain via lowering of iNOS.

Although studies mentioned above have demonstrated the association of iNOS and the role of its inhibition in the improvement of neuropathic pain in animals, the best of our knowledge, this study is showing the association of DPNP and iNOS serum level in human for the first time. Besides, the effect of PGB in DPNP via iNOS is also a novelty of the current study, which can be a new approach to treat neuropathic pain.

\section{Conclusion}

This study revealed a direct correlation between the reduction of iNOS and thus the decrement in NO production with the improvement of DPNP after PGB usage. Therefore we can conclude that the painrelieving properties of PGB would be through the iNOS, NO, pathway. According to our study, this palliative effect of PGB on DPNP may protect the degeneration of the peripheral and central nervous system by probable direct or indirect inhibition of iNOS. Thus the iNOS inhibitors can be a novel approach for the treatment of neuropathic pain. Additional cellular, animal, and human studies are needed to clarify this particular effect.

\section{Abbreviations}

\section{ADRs}

Adverse drug reactions

BMI

Body Mass Index 
BT

Before starting the treatment

CCl

Chronic constriction injury

CONSORT

Consolidated Standards of Reporting Trials

DN

Diabetic Neuropathy

DPNP

Diabetic Peripheral Neuropathic Pain

\section{ELISA}

Enzyme-linked immune sorbent assay

FBS

Fasting Blood Sugar

FDA

Food and Drug Administration

HA1c

Hemoglobin A1c;

iNOS

inducible Nitric Oxide Synthase

L-NAME

$N^{G}$-nitro-L-Arginine Methyl Ester

L-NMMA

$\mathrm{N}^{\mathrm{G}}$-monomethyl-L-arginine

\section{MNSI}

Michigan Neuropathy Screening Instrument

NO

Nitric Oxide

OMT

One month after the treatment

PGB

Pregabalin

TMT

Two months after the treatment

VAS

Visual Analogue Scale

Declarations

Ethics approval and consent to participate 
This study was approved by the ethics committee of Shahid Beheshti University of Medical Sciences, SBUMS, Tehran, Iran (ethical code: IR.SBMU.MSP.REC.1395.41) and registered in the Iranian Registry of Clinical Trials website: http://www.irct.ir (at 14/03/2016) and approved in 19/07/2017, the registration number is: (IRCT2017012932277N1). The study was conducted in the Diabetes Clinic of Shohada Hospital at SBUMS on 22/07/2017 till 18/03/2018. We have explained the study objectives and protocols to the subjects and obtained written informed consent before they participated in the study.

\section{Consent for publication}

Not applicable.

\section{Availability of data and material}

All data generated or analyzed during this study are included in this published article.The datasets are available from the corresponding author on a reasonable request.

\section{Competing interests}

The authors declare that they have no competing interests.

\section{Funding}

The present article was financially supported by the "Research Department of the School of Medicine of Shahid Beheshti University of Medical Sciences" (Grant No: 11622).

\section{Authors' Contributions}

Khojasteh Joharchi: Main supervisor and manager of the whole project, acquisition, conception and design of the work, monitoring all affairs, checking the laboratory works and data, revising the manuscript. MoosarezaMemari: Monitoring the patients, collecting the data, and doing the main work as part of his Ph.D. thesis, and also drafting the article. Mir Saber SadaatAmini: The endocrinologist, visiting and examining the patients. YaldaKhazaeiPoul: lab technician.

\section{Acknowledgments}

The authors would like to appreciate the cooperation of all patients, and the staffs of the diabetes clinic of Shohada Hospital, involved in this project. We also have to express gratitude to doctorEznollahAzargashb for his friendly assistance on statistical analyzes of the data.

\section{Authors' Information}

MoosarezaMemari:

Pharm D, Ph.D. of Pharmacology, Department of Clinical Pharmacology, Bouali Hospital, School of Medicine, Ardabil University of Medical Sciences (ARUMS), Ardebil, Iran, and Department of 
Pharmacology, School of Medicine, Shahid Beheshti University of Medical Sciences, Tehran, Iran.

Mir Saber SadaatAmini:

MD, Endocrinologist, Assistant Professor, Diabetes Clinic, Shohada Hospital,School of Medicine, Shahid Beheshti University of Medical Sciences, Tehran, Iran

YaldaKhazaeiPoul:

M.S., Lab technician, Department of Pharmacology, School of Medicine, Shahid Beheshti University of Medical Sciences, Tehran, Iran

Khojasteh Joharchi:

MD., Ph.D., Associate Professor of Pharmacology, Department of Pharmacology, School of Medicine, Shahid Beheshti University of Medical Sciences, Tehran, Iran

\section{References}

1. Purwata TE. High TNF-alpha plasma levels and macrophages iNOS and TNF-alpha expression as risk factors for painful diabetic neuropathy. Journal of pain research. 2011;4:169.

2. Ahlawat A, Rana A, Goyal N, Sharma S. Potential role of nitric oxide synthase isoforms in pathophysiology of neuropathic pain. Inflammopharmacology. 2014;22(5):269-78.

3. Obrosova IG, Mabley JG, Zsengellér Z, Charniauskaya T, Abatan Ol, Groves JT, et al. Role for nitrosative stress in diabetic neuropathy: evidence from studies with a peroxynitrite decomposition catalyst. The FASEB journal. 2005;19(3):401-3.

4. Sifuentes-Franco S, Pacheco-Moisés FP, Rodríguez-Carrizalez AD, Miranda-Díaz AG. The Role of Oxidative Stress, Mitochondrial Function, and Autophagy in Diabetic Polyneuropathy. Journal of diabetes research. 2017;2017.

5. Miclescu A, Gordh T. Nitric oxide and pain:'Something old, something new'. Acta Anaesthesiologica Scandinavica. 2009;53(9):1107-20.

6. Wilcox GL. Spinal mediators of nociceptive neurotransmission and hyperalgesia: relationships among synaptic plasticity, analgesic tolerance, and blood flow. APS Journal. 1993;2(4):265-75.

7. Gorąca A, Huk-Kolega H, Piechota A, Kleniewska P, Ciejka E, Skibska B. Lipoic acid-biological activity and therapeutic potential. Pharmacological Reports. 2011;63(4):849-58.

8. Mukherjee P, Cinelli MA, Kang S, Silverman RB. Development of nitric oxide synthase inhibitors for neurodegeneration and neuropathic pain. Chemical Society reviews. 2014;43(19):6814-38.

9. Jean YH, Chen WF, Sung CS, Duh CY, Huang SY, Lin CS, et al. Capnellene, a natural marine compound derived from soft coral, attenuates chronic constriction injury-induced neuropathic pain in rats. British journal of pharmacology. 2009;158(3):713-25. 
10. Bonnefous C, Payne JE, Roppe J, Zhuang H, Chen X, Symons KT, et al. Discovery of inducible nitric oxide synthase (iNOS) inhibitor development candidate KD7332, part 1: Identification of a novel, potent, and selective series of quinolinone iNOS dimerization inhibitors that are orally active in rodent pain models. Journal of medicinal chemistry. 2009;52(9):3047-62.

11. Kwiatkowski K, Piotrowska A, Rojewska E, Makuch W, Mika J. The RS504393 influences the level of nociceptive factors and enhances opioid analgesic potency in neuropathic rats. Journal of Neuroimmune Pharmacology. 2017;12(3):402-19.

12. Tesfaye S, Boulton AJ, Dickenson AH. Mechanisms and management of diabetic painful distal symmetrical polyneuropathy. Diabetes care. 2013;36(9):2456-65.

13. Juster-Switlyk K, Smith AG. Updates in diabetic peripheral neuropathy. F1000Research. 2016;5.

14. Bajaj S. RSSDI clinical practice recommendations for the management of type 2 diabetes mellitus 2017. International journal of diabetes in developing countries. 2018;38(1):1-115.

15. Verma V, Singh N, Singh Jaggi A. Pregabalin in neuropathic pain: evidences and possible mechanisms. Current neuropharmacology. 2014;12(1):44-56.

16. Pelletier JP, Jovanovic D, Fernandes JC, Manning P, Connor JR, Currie MG, et al. Reduced progression of experimental osteoarthritis in vivo by selective inhibition of inducible nitric oxide synthase. Arthritis \& Rheumatism. 1998;41(7):1275-86.

17. Song Y, Jun J-H, Shin E-J, Kwak Y-L, Shin J-S, Shim J-K. Effect of pregabalin administration upon reperfusion in a rat model of hyperglycemic stroke: Mechanistic insights associated with highmobility group box 1. PloS one. 2017;12(2):e0171147.

18. Amany S, Heba K. Effect of pregabalin on erectile function and penile NOS expression in rats with streptozotocin-induced diabetes. Experimental and Clinical Endocrinology \& Diabetes. 2013;121(04):230-3.

19. Tufekci A, Koyuncuoglu HR, Kirbas S, Yilmaz HR, Kirbas A. The effects of pregabalin on cerebral cortical oxidative stress of rats on pentylenetetrazole induced epileptic seizure/Sicanlarda pentilentetrazol ile olusturulan epileptik nobet modelinde pregabalinin beyin korteksinde oksidatif stres uzerine etkileri. Epilepsi: Journal of the Turkish Epilepsi Society. 2013;19(1):7-15.

20. Joharchi K, Memari M, Azargashb E, Saadat N. Efficacy and safety of duloxetine and Pregabalin in Iranian patients with diabetic peripheral neuropathic pain: a double-blind, randomized clinical trial. Journal of Diabetes \& Metabolic Disorders. 2019;18(2):575-82.

21. McCulloch DK, Hayward RA. Screening for type 2 diabetes mellitus. UpToDate UpToDate. 2016.

22. Herman W, Pop-Busui R, Braffett B, Martin C, Cleary P, Albers J, et al. Use of the Michigan Neuropathy Screening Instrument as a measure of distal symmetrical peripheral neuropathy in Type 1 diabetes: results from the Diabetes Control and Complications Trial/Epidemiology of Diabetes Interventions and Complications. Diabetic Medicine. 2012;29(7):937-44.

23. Heller GZ, Manuguerra M, Chow R. How to analyze the Visual Analogue Scale: Myths, truths, and clinical relevance. Scandinavian journal of pain. 2016;13:67-75.

24. Fornasari D. Pharmacotherapy for Neuropathic Pain: A Review. Pain and therapy. 2017;6(1):25-33. 
25. Javed S, Petropoulos IN, Alam U, Malik RA. Treatment of painful diabetic neuropathy. Therapeutic advances in chronic disease. 2015;6(1):15-28.

26. Joharchi $K$, Jorjani $M$. The role of nitric oxide in diabetes-induced changes of morphine tolerance in rats. European journal of pharmacology. 2007;570(1-3):66-71.

27. Mortada Y, Khojasteh K, Zarei M, Mansouri A, Jorjani M. How nitric oxide increases in diabetic morphine tolerated male rats. Iranian Journal of pharmaceutical research: IJPR. 2017;16(2):630.

28. Arias-Salvatierra D, Silbergeld EK, Acosta-Saavedra LC, Calderon-Aranda ES. Role of nitric oxide produced by iNOS through the NF-KB pathway in migration of cerebellar granule neurons induced by lipopolysaccharide. Cellular signaling. 2011;23(2):425-35.

29. Kumar A, Chen S-H, Kadiiska MB, Hong J-S, Zielonka J, Kalyanaraman B, et al. Inducible nitric oxide synthase is key to peroxynitrite-mediated, LPS-induced protein radical formation in murine microglial BV2 cells. Free Radical Biology and Medicine. 2014;73:51-9.

30. Ahlawat A, Sharma S. A new promising simultaneous approach for attenuating type ll diabetes mellitus induced neuropathic pain in rats: iNOS inhibition and neuroregeneration. European journal of pharmacology. 2018;818:419-28.

31. Sasaki A, Mabuchi T, Serizawa K, Takasaki I, Andoh T, Shiraki K, et al. Different roles of nitric oxide synthase-1 and-2 between herpetic and postherpetic allodynia in mice. Neuroscience. 2007;150(2):459-66.

32. Wang C, Wang C. Anti-nociceptive and anti-inflammatory actions of sulforaphane in chronic constriction injury-induced neuropathic pain mice. Inflammopharmacology. 2017;25(1):99-106.

33. Xu J, Kim G-M, Chen S, Yan P, Ahmed SH, Ku G, et al. iNOS and nitrotyrosine expression after spinal cord injury. Journal of neurotrauma. 2001;18(5):523-32.

34. Dauch JR, Yanik BM, Hsieh W, Oh SS, Cheng HT. Neuron-astrocyte signaling network in spinal cord dorsal horn mediates painful neuropathy of type 2 diabetes. Glia. 2012;60(9):1301-15.

35. Yang Y, Zhang J, Liu Y, Zheng Y, Bo J, Zhou X, et al. Role of nitric oxide synthase in the development of bone cancer pain and effect of L-NMMA. Molecular medicine reports. 2016;13(2):1220-6.

36. Sung C-S, Wen Z-H, Chang W-K, Ho S-T, Tsai S-K, Chang Y-C, et al. Intrathecal interleukin-1 $\beta$ administration induces thermal hyperalgesia by activating inducible nitric oxide synthase expression in the rat spinal cord. Brain research. 2004;1015(1-2):145-53.

\section{Figures}




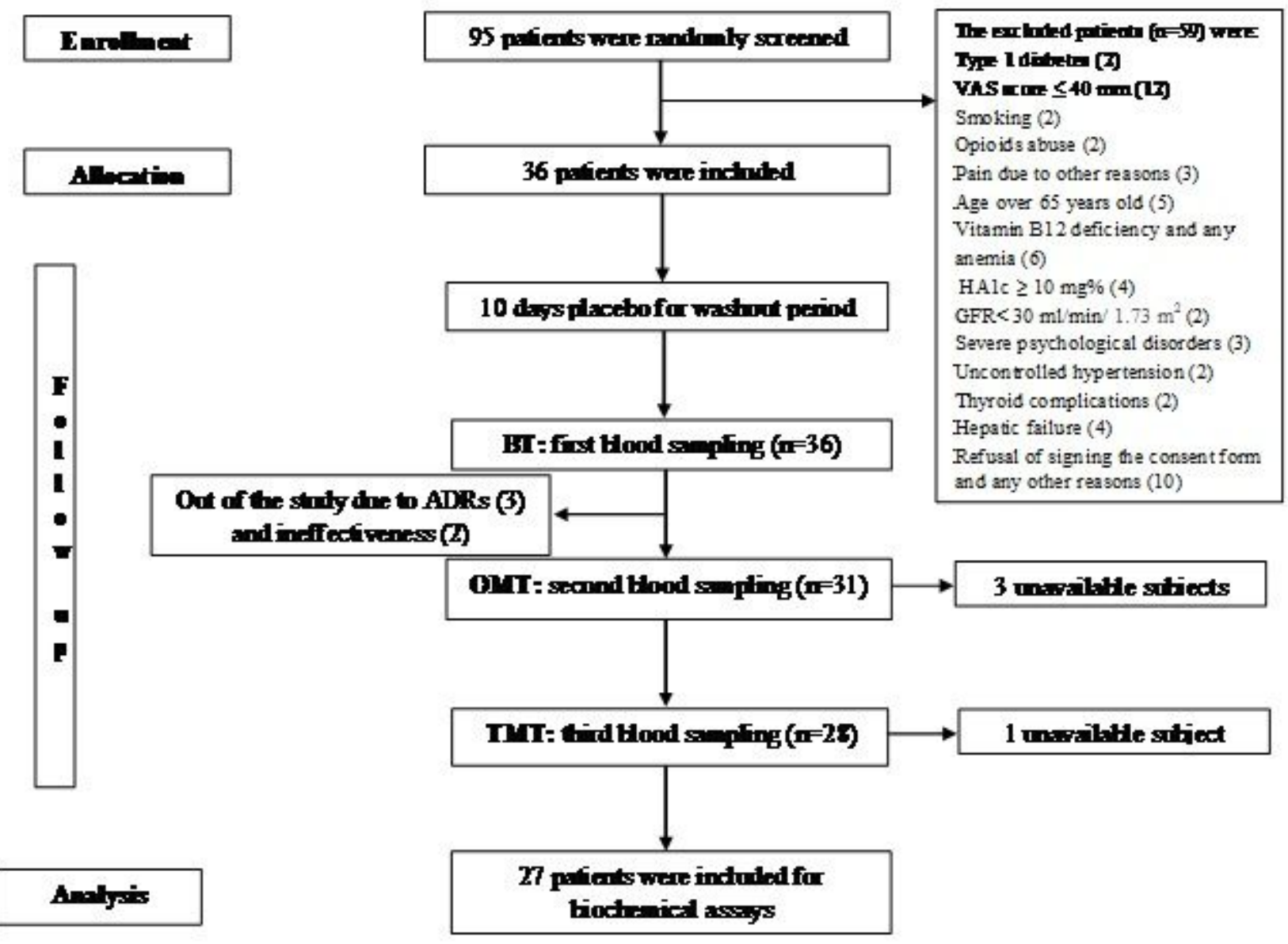

Figure 1

The study CONSORT flow chart: n: number, BT: Before starting the Treatment, ADRs: Adverse Drug Reactions, OMT: One Month (4 weeks) after Treatment, TMT: Two Months (8 weeks) after Treatment.

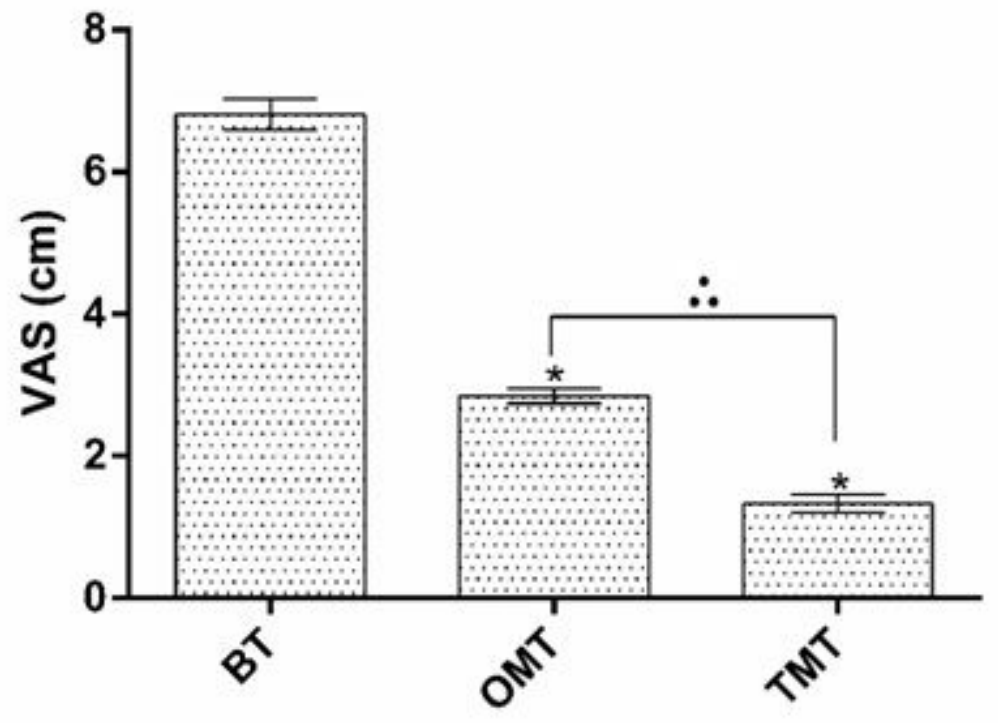

Figure 2 
Mean pain scores during the study period; $X$-axis represents the study time points; $Y$-axis represents the pain scores in the Visual Analog Scale (VAS) in centimeters (cm); BT: Before treatment; OMT: One Month after Treatment; TMT: Two Months after Treatment. Data analysis was done by Repeated Measure Test and are presented as Mean $\pm S E$, * $P$-value $<0.05$ vs. baseline; $₫ P$-value $<0.05$ TMT vs. OMT.

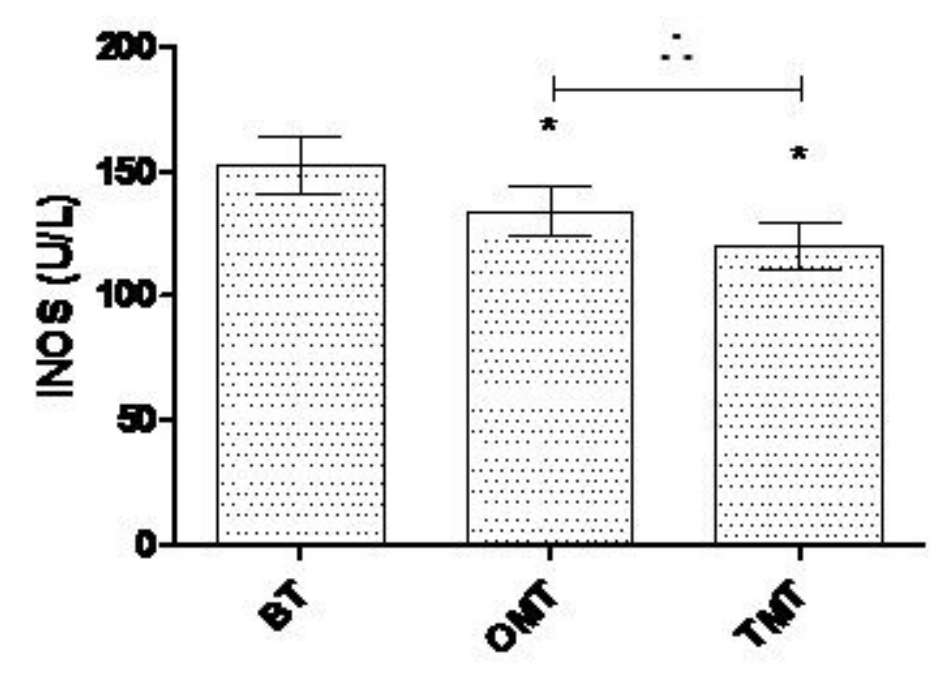

\section{Figure 3}

The iNOS serum levels during the study period: iNOS: inducible nitric oxide synthase, $X$-axis represents the study timepoints; Y-axis represents the iNOS serum levels in Unit/Liter (U/L); BT: Before Treatment; OMT: One Month after Treatment; TMT: Two Months after Treatment. Data analysis was done by Repeated Measure Test and are presented as Mean $\pm S E ; *$-value $<0.05$ vs. baseline; $\otimes P$-value $<0.05, T M T$ vs. OMT.

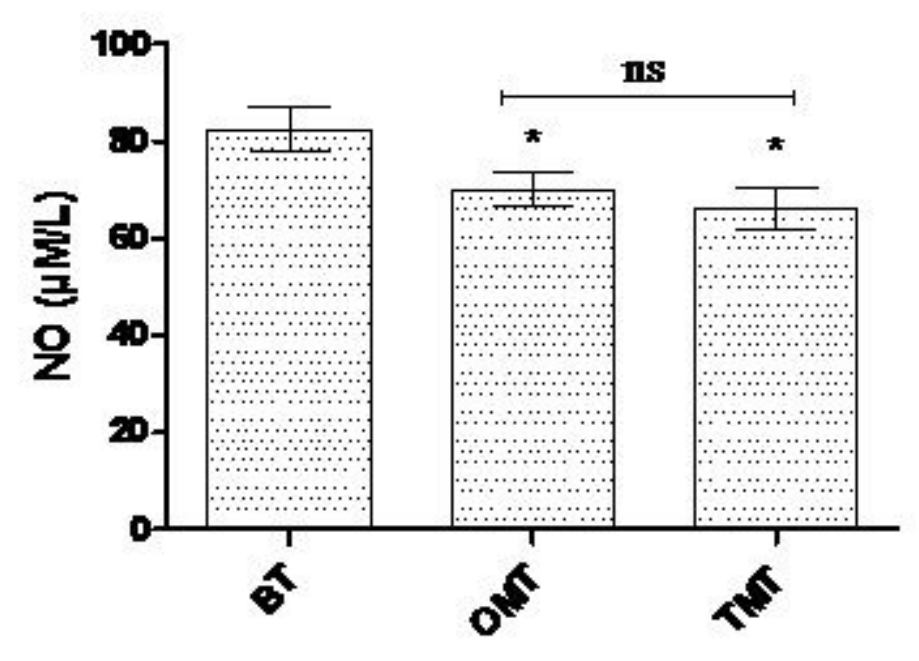

Figure 4

The serum levels of NO during the study period. NO: nitric oxide; X-axis represents the study time points; $\mathrm{Y}$-axis represents the NO serum levels in micromole/Liter ( $\square \mathrm{M} / \mathrm{L})$; BT: Before Treatment; OMT: One Month 
after Treatment; TMT: Two Months after Treatment. Data analysis was done by Repeated Measure Test and are presented as Mean \pm SE. * P-value< 0.05 vs. baseline; ns: not significant(P-value=0.091)TMT vs. OMT. 